\title{
Inverse Cutting of Posterior Lamellar Corneal Grafts by a Femtosecond Laser
}

\author{
Jesper Hjortdal ${ }^{*}$, Esben Nielsen, Anders Vestergaard and Anders Søndergaard
}

Department of Ophthalmology, Aarhus University Hospital, Nørrebrogade 44, 8000 Aarhus C, Denmark

\begin{abstract}
Purpose: Posterior lamellar grafting of the cornea has become the preferred technique for treatment of corneal endothelial dysfunction. Posterior lamellar grafts are usually cut by a micro-keratome or a femto-second laser after the epithelial side of the donor cornea has been applanated. This approach often results in variable central graft thickness in different grafts and an increase in graft thickness towards the periphery in every graft. The purpose of this study was to evaluate if posterior lamellar grafts can be prepared from the endothelial side by a femto-second laser, resulting in reproducible, thin grafts of even thickness.
\end{abstract}

Methods: A CZM $500 \mathrm{kHz}$ Visumax femto-second laser was used. Organ cultured donor grafts were mounted in an artifical anterior chamber with the endothelial side up and out. Posterior grafts of $7.8 \mathrm{~mm}$ diameter and 130 micron thickness were prepared by femto-second laser cutting. A standard DSAEK procedure was performed in 10 patients with Fuchs endothelial dystrophy. Patients were followed-up regularly and evaluated by measurement of complications, visual acuity, corneal thickness (Pentacam HR), and endothelial cell density.

Results: Femto-laser cutting of grafts and surgery was uncomplicated. Rebubbling was necessary in 5 of 10 cases (normally only in 1 of 20 cases). All grafts were attached and cleared up during the first few weeks. After six months, the average visual acuity was 0.30 (range: 0.16 to 0.50 ), corneal thickness was $0.58 \mathrm{~mm}$ (range 0.51 to 0.63 ), and endothelial cell density was 1.570 per sq. $\mathrm{mm}$ (range: 1.400 to 2.000 cells per sq. $\mathrm{mm}$ ). The grafts were of uniform thickness, but substantial interface haze was present in most grafts.

Conclusions: Posterior lamellar corneal grafts can be prepared from the endothelial side using a femto-second laser. All grafts were clear after 6 months with satisfying endothelial cell counts. Poor visual acuity caused by interface scatter was observed in most patients. Femto-second laser cutting parameters needs to be optimised to enable smooth cutting in the posterior stroma.

Keywords: Corneal grafts, Femto-laser, cornea, DSAEK.

\section{INTRODUCTION}

Posterior lamellar keratoplasty, most often performed as Descemet's Stripping Automated Endothelial Keratoplasty (DSAEK), has become the standard treatment for patients with symptomatic Fuchs' endothelial dystrophy and secondary bullous keratopathy.

The donor lamellar graft is prepared by removing most of the anterior corneal stroma by a mechanical microkeratome either in the eye bank or by the surgeon immediately before surgery. The resulting posterior lamellar grafts often vary considerably in thickness and are typically thicker at the periphery than in the centre. As graft thickness significantly may influence the final visual acuity in the recipient [1], it would be preferable to improve the cutting technique to ensure that the donor grafts are always prepared very thin. The negative optical power of microkeratome prepared grafts also results in a small, but significant hyperopic shift in the refractive power of the recipient [2]. This could be avoided if the donor grafts were prepared to have even thinness.

*Address correspondence to this author at the Department of Ophthalmology, Aarhus University Hospital, Nørrebrogade 44, 8000 Aarhus C, Denmark; Tel: +45 78463221; Fax: +45 86121653;

E-mail: jesper.hjortdal@dadlnet.dk
Femtosecond lasers are widely and successfully used for preparation of flaps in LASIK. In a prospective randomized clinical study, however, patients receiving a femtosecond laser prepared posterior lamellar graft obtained lower visual acuity compared with patients undergoing penetrating keratoplasty [3]. In the study, low-frequency, high-energy femtosecond laser cutting was done with a flat applicator plate applied on the epithelial side of the donor eye. This may result in distortion of the deeper layers of the donor cornea, eventually resulting in a rough cut-surface [4].

In the present case-series, we used a fast, low-energy femtosecond laser with a concave applicator plate applied on the posterior, endothelial side of inverted human corneal donor tissue to prepare posterior lamellar grafts (Fig. 1). With this technique, it should be possible to prepare plane and thin posterior lamellar grafts.

\section{METHODS AND SUBJECTS}

Ten patients with Fuchs' endothelial dystrophy underwent standard posterior lamellar keratoplasty with implantation of a femtosecond laser prepared posterior lamellar graft. In accordance with Danish law, informed oral consent was obtained from all patients. Organ cultured donor grafts with an endothelial cell density above 2.000 cells $/ \mathrm{mm}^{2}$ 
A

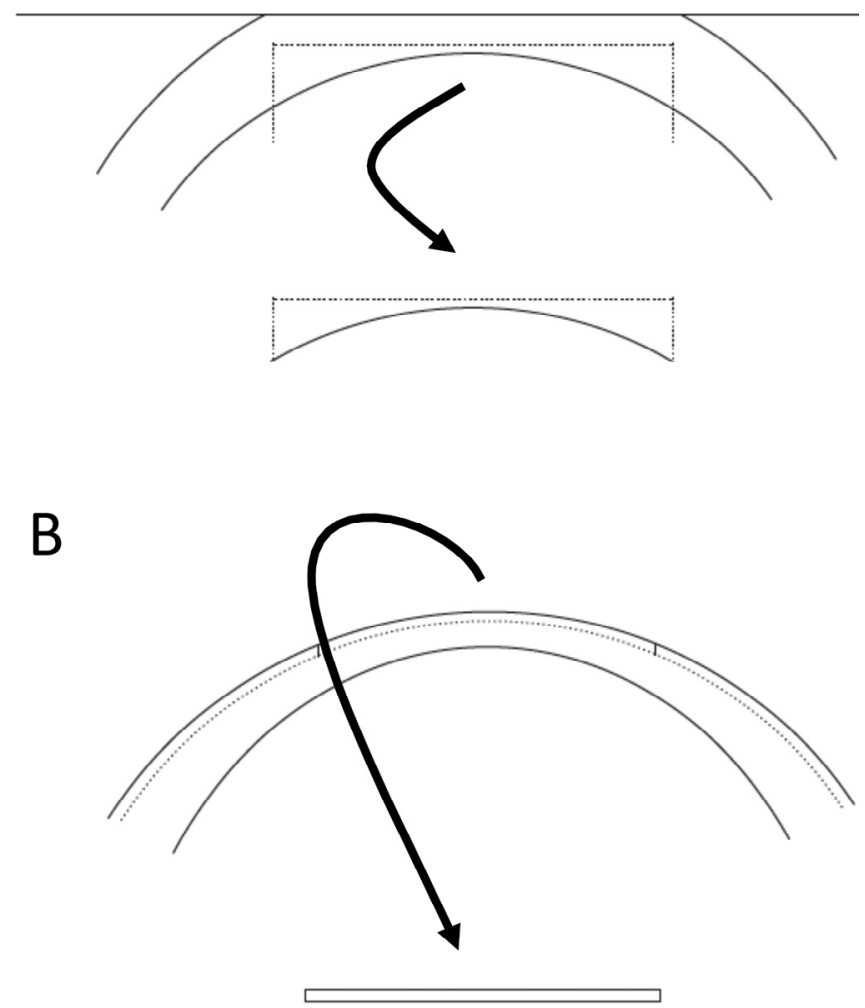

Fig. (1). (A) Schematic drawing of posterior lamellar graft preparation with a microkeratome or femtosecond laser with flat applanation of the epithelial side creation of a donor cornea. (B) Schematic drawing of posterior lamellar graft with a femtosecond laser with curved applanation of the endothelial side of a donor cornea.

were delivered by The Danish Cornea Bank, Aarhus University Hospital. After 24 hours storage in transport medium with 8\% Dextrane, the donor cornea buttons were mounted inversely (endothelial side outwards) in an artificial anterior chamber (Moria, Antony, France) and pressurised with approximately $20 \mathrm{~cm}$ of isotonic saline. A few drops of organ culture medium were regularly applied to the endothelium. A $500 \mathrm{kHz}$ Visumax femtosecond laser (Carl Zeiss Meditec, Jena, Germany) was used for cutting the donor lamella. After mounting the concave applicator glass in the laser, the corneal donor tissue was centred and slightly applanated to ensure contact between the applicator and the corneal endothelium covered with a thin layer of organ culture medium (Fig. 2). The graft was prepared to a $7.8 \mathrm{~mm}$ diameter and a thickness of 130 micrometre. After laser cutting, the graft was gently loosened and kept in organ culture medium until implantation approximately 30 minutes later.

The standard surgical technique for posterior lamellar transplantation was used. A superior $4 \mathrm{~mm}$ corneo-scleral tunnel was created and a chamber maintainer inserted at 3 o'clock. Descemets membrane was removed in a $7.5 \mathrm{~mm}$ diameter zone, and using a Busin spatula and forceps (Moria, Antony, France) the graft was pulled into the anterior chamber. The graft was centred and the anterior chamber filled with a large air-bubble. After 2 hours, the air-bubble was reduced to approximately $40 \%$ of the chamber height. In 5 of the 10 patients phacoemulsification with IOL implantation was done in combination with posterior lamellar transplantation.

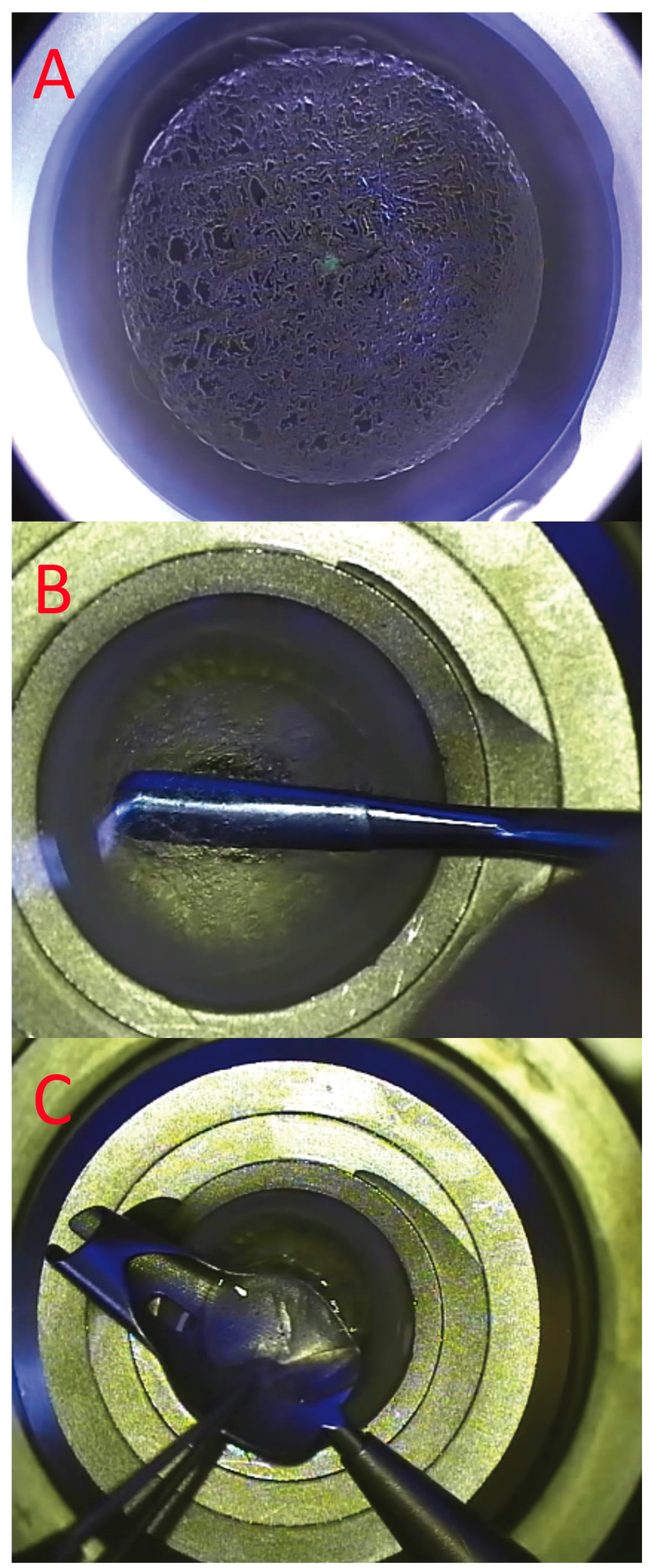

Fig. (2). Femtosecond laser cutting of an inverted donor cornea. (A) Plasma bubbles are seen immediately after cutting. (B) Remaining tissue bridges are broken. (C) Graft is positioned in glide before implantation. 
Patients were routinely treated with Tobradex eye drops 6 times daily for one week, then 4 times daily for 2 months and tapering of to a stop after approximately 8 months. Patients were seen daily the first week, weekly the first month, and then every 3 months. Clinical follow-up included measurement of visual acuity, slit-lamp examination, tonometry, and Pentacam tomography (Oculus, Wetzlar, Germany) and specular microscopy (Topcon SP 1000, Tokyo, Japan) after 12 months.

\section{RESULTS}

During the first 3 days, re-bubbling with air was necessary in 5 of the 10 patients due to complete or partial graft detachment. After one week all grafts were attached and the corneal oedema had started to clear. The follow-up period was uneventful with no episodes of graft rejection in any patient.

Fig. (3A) shows the changes in central corneal thickness during the first year. As indicated by the corneal thickness, all corneas appeared normo-hydrated and clear after one month.

At the 12-month follow-up visit the average endothelial cell density was 1.779 cells $/ \mathrm{mm}^{2}$, (range 1.200 to 2.300 ).

In Fig. (3B) the development in best spectacle corrected visual acuity is shown. A small increase is seen from one month to 12-month after surgery, but only one of the 10 patients had obtained 20/40 or better at the 12-month visit.

\section{A}

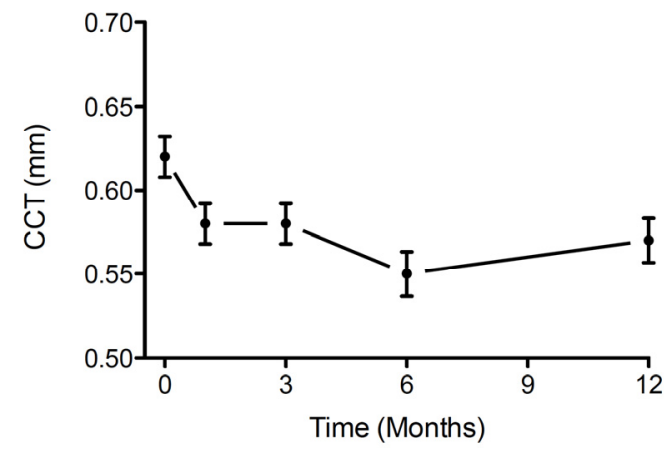

B

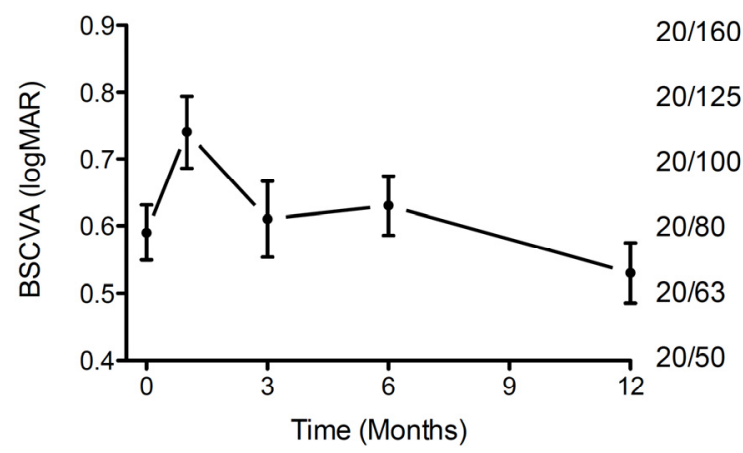

Fig. (3). Central corneal thickness (part A) and best spectacle corrected visual acuity (logMAR left, Snellen lines right) (part B) before ( 0$)$ and after implantation of femtosecond laser prepared posterior lamellar grafts. Mean +/- SEM of 10 patients.
Slit-lamp and Pentacam tomography Scheimpflug images showed that the posterior lamellar grafts were well attached and of even thickness. In all patients, however, a fairly dense interface scatter was visible (Fig. 4).

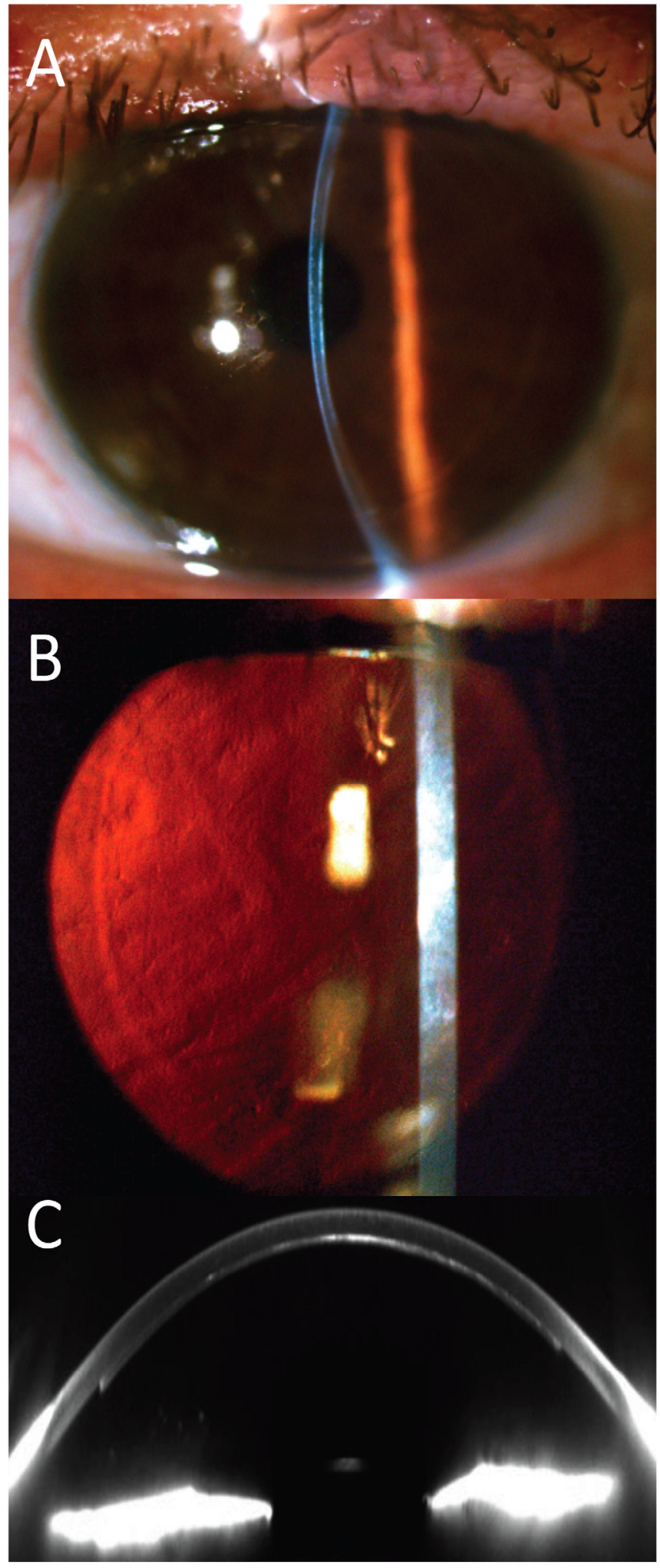

Fig. (4). Clinical pictures 6 months after implantation of a femtosecond laser prepared posterior lamellar graft. Interface haze is visible in (A) slit-lamp photo, (B) slit-lamp photo with retroillumination, and in (C) Pentacam Scheimpflug picture. 
At the 12-month visit, patients with no benefit of the operation were offered retransplantation with a microkeratome prepared donor graft. Three patients accepted the offer and were regrafted. After 3 months, all regrafted patients had improved in best spectacle corrected visual acuity.

\section{DISCUSSION}

The present case-series demonstrate that femtosecond laser cutting from the endothelial side can prepare plane and thin posterior lamellar grafts. Endothelial cell density after one year was similar to endothelial cell density after one year in patients undergoing DSAEK with microkeratome prepared grafts [5]. However, interface haze significantly negatively affected the visual outcome of the procedure even when a fast, low-energy, non-applanating femtosecond laser is used. It may be speculated that the parallel organization of the collagen fibrils in the posterior part of the stroma generate tiny collagen fibril strands when the tissue is cut during femtosecond laser generated plasma formation.

Further refinements of laser settings or in donor cornea preparation are necessary before femtosecond lasers routinely can be used for preparation of posterior lamellar grafts.

\section{ACKNOWLEDGEMENT}

Declared none.

\section{CONFLICT OF INTEREST}

Declared none.

\section{REFERENCES}

[1] Chen ES, Terry MA, Shamie N, et al. Descemet-stripping automated endothelial keratoplasty: six-month results in a prospective study of 100 eyes. Cornea 2008; 27: 514-20.

[2] Jun B, Kuo AN, Afshari NA, et al. Refractive change after Descemet stripping automated endothelial keratoplasty surgery and its correlation with graft thickness and diameter. Cornea 2009; 28: 19-23.

[3] Cheng YY, Schouten JS, Tahzib NG, et al. Efficacy and safety of femtosecond laser-assisted corneal endothelial keratoplasty: a randomized multicenter clinical trial. Transplantation 2009; 88(11): 1294-302.

[4] Mootha VV, Heck E, Verity SM, et al. Comparative study of descemet stripping automated endothelial keratoplasty donor preparation by moria cbm microkeratome, horizon microkeratome, and intralase FS60. Cornea 2011; 30: 320-4.

[5] Hjortdal J, Ehlers N. Descemet's stripping automated endothelial keratoplasty and penetrating keratoplasty for Fuchs' endothelial dystrophy. Acta Ophthalmol 2009; 87(3): 310-4. 